\title{
The Effect of the Singular Potential Functions for Eigenvalues of Sturm-L1ouville Operators
}

\author{
Bülent Y1lmaz
}

(Communicated by Murat TOSUN)

\begin{abstract}
In this article we obtain asymptotic formulas of arbitrary order for eigenfunctions and eigenvalues of the non-self adjoint Sturm-Liouville operators with Dirichlet boundary conditions, when the potential is a summable function. Then using these we compute the main part of the eigenvalues in special cases. The eigenvalues obtained by the asymptotic method and the eigenvalues obtained by the finite difference method followed by a numerical correction, are compared.
\end{abstract}

Keywords: Asymptotic formulas, Sturm-Liouville operators.

AMS Subject Classification (2010): Primary: 47E05.

${ }^{*}$ Corresponding author

\section{Introduction}

Let $L(q)$ be an operator generated in $L_{2}[0,1]$ by the expression

$$
L(q)=-y^{\prime \prime}(x)+q(x) y(x), \quad 0 \leq x \leq 1
$$

and by Dirichlet boundary conditions

$$
y(0)=y(1)=0,
$$

where $q(x)$ is a complex-valued summable function.

In this article, we consider the small and large eigenvalues of the operator $L(q)$ when $q(x)$ has a singularity. The large eigenvalues are investigated by the asymptotic method given in [1,2]. Note that in classical investigations in order to obtain the asymptotic formulas of order $O\left(n^{-l}\right)$ it is required that $q(x)$ be $(l-1)$ times differentiable $[4,11]$. The method of [1] gives the possibility of obtaining the asymptotic formulas of order $O\left(n^{-l}\right)$ of eigenvalues and eigenfunctions of $L(q)$ when $q(x)$ is arbitrary summable complex- valued function. The eigenvalues are compared by asymptotic method and corrected approximation.

Expression of differential equations in matrix form and the advances in the field of the computers have led to major developments in numerical methods. Regarding the numerical solution of the Sturm-Liouville problems, Finite Difference method is amongst the popular methods. Finite Difference method can give effective results

Received : 05-August-2015, Accepted : 14-December-2016

This article is the written version of author's plenary talk delivered on September 03-August 31, 2015 at 4th International Eurasian Conference on Mathematical Sciences and Applications (IECMSA-2015) in Athens, Greece. 
for the eigenvalues when it is used in connection with asymptotic correction technique. In the paper [13] the Sturm-Liouville problems with Dirichlet and the general boundary conditions were studied respectively. Paine found the correction of Finite Difference Eigenvalue Approximations for Sturm-Liouville Problems. Chen [16] used the differential transform method to solve the eigenvalue problems. Ghelardoni and Gheri [18] used the shooting technique for the calculation of the eigenvalues of Sturm-Liouville problem by considering the Prüfer transformation given in [19].

\section{Asymptotic Formulas For Eigenvalues}

It is well-known [7] that the eigenvalues of the operator $L(q)$ consists of the sequences $\left\{\lambda_{n}\right\}$ satisfying

$$
\lambda_{n}=(n \pi)^{2}+O(1)
$$

for $n \geq N$. Here and in forthcoming relations we denote by $N$ a large positive integer, that is, $N \gg 1$. From these formulas one can easily obtain the following inequalities from which we use essentially:

$$
\left|\lambda_{n}-(\pi k)^{2}\right|>|(n-k) \pi||(n+k) \pi|-c_{1} n^{\frac{1}{2}}>c_{2} n, \forall k \neq n, k=0,1, \ldots,
$$

for $n \geq N$, where we denote by $c_{m}$, for $m=1,2, \ldots$, the positive constants whose exact value are inessential. To obtain the asymptotic formula for eigenvalues $\lambda_{n}$ and corresponding normalized eigenfunctions $\Phi_{n}(x)$ of $L(q)$ we use (4) and the following well-known relation

$$
\left(\lambda_{N}-(\pi n)^{2}\right)\left(\Phi_{N}(x), \sin n \pi x\right)=\left(q(x) \Phi_{N}(x), \sin n \pi x\right)
$$

Arguing as in the proof of Lemma 1 of [2], and using (5) and the decomposition

$$
\Phi_{N}(x)=\sum_{n_{1}>-n}^{\infty} 2\left(\Phi_{N}(x), \sin \left(n+n_{1}\right) \pi x\right) \sin \left(n+n_{1}\right) \pi x
$$

of $\Phi_{N}(x)$ by orthonormal basis $\left\{\sqrt{2} \sin \left(n+n_{1}\right) \pi x: n_{1}>-n\right\}$ we get

$$
\begin{gathered}
\left(q(x) \Phi_{N}(x), \sin n \pi x\right)= \\
\sum_{n_{1}>-n}^{\infty} 2\left(q(x),\left(\sin \left(n+n_{1}\right) \pi x\right)(\sin n \pi x)\right)\left(\Phi_{N}(x), \sin \left(n+n_{1}\right) \pi x\right)
\end{gathered}
$$

from which using the multiplication formula in (7) and then replacing $2 n+n_{1}$ by $k$, we get

$$
\begin{aligned}
\left(q(x) \Phi_{N}(x), \sin n \pi x\right) & =\sum_{n_{1}=1}^{\infty} C_{n_{1}}\left(\Phi_{N}(x), \sin \left(n+n_{1}\right) \pi x\right) \\
& +\sum_{n_{1}=1}^{\infty} C_{n_{1}}\left(\Phi_{N}(x), \sin \left(n-n_{1}\right) \pi x\right)
\end{aligned}
$$

substituting this result in to (5) we obtain

$$
\left(\lambda_{N}-(\pi n)^{2}\right)\left(\Phi_{N}(x), \sin n \pi x\right)=\sum_{n_{1}=-\infty}^{\infty} C_{n_{1}}\left(\Phi_{N}(x), \sin \left(n+n_{1}\right) \pi x\right)
$$

where $C_{n}=\int_{0}^{1} q(x) \cos n \pi x d x$ and, without loss of generality, we assume that $C_{0}=0$. Note that $C_{n} \rightarrow 0, C_{n}=C_{-n}$, as $|n| \rightarrow \infty$

Now we isolate the terms at right side of (9), containing $\left(\Phi_{n}(x), \sin n \pi x\right)$ (i.e., the case $\left.n_{1}=-2 n\right)$, Using the equality

$$
\left(\Phi_{n}(x), \sin \left(n+n_{1}\right) \pi x\right)=\sum_{\substack{n_{2}=-\infty \\ n_{1} \neq-2 n}}^{\infty} \frac{C_{n_{2}}\left(\Phi_{n}(x), \sin \left(n+n_{1}+n_{2}\right) \pi x\right)}{\lambda_{n}-\left(\pi\left(n+n_{1}\right)\right)^{2}}
$$


which can be obtained from (9) by taking $n$ and $n+n_{1}$ instead of $N$ and $n$, we arrive at

$$
\begin{gathered}
\left(\lambda_{n}-(\pi n)^{2}\right)\left(\Phi_{n}(x), \sin n \pi x\right)=-C_{2 n}\left(\Phi_{n}(x), \sin n \pi x\right) \\
\quad+\sum_{\substack{n_{1}, n_{2}=-\infty \\
n_{1} \neq-2 n}}^{\infty} \frac{C_{n_{1}} C_{n_{2}}\left(\Phi_{n}(x), \sin \left(n+n_{1}+n_{2}\right) \pi x\right)}{\lambda_{n}-\left(\pi\left(n+n_{1}\right)\right)^{2}}
\end{gathered}
$$

Again we isolate the terms for $n_{1}+n_{2}=0,-2 n$ from the summation and use the equality $C_{n_{1}}=C_{-n_{1}}$ to obtain

$$
\begin{gathered}
\left(\lambda_{n}-(\pi n)^{2}\right)\left(\Phi_{n}(x), \sin n \pi x\right)=\left(\Phi_{n}(x), \sin n \pi x\right)\left\{-C_{2 n}\right. \\
\left.+\sum_{\substack{n_{1}=-\infty \\
n_{1} \neq 0,-2 n}}^{\infty} \frac{C_{n_{1}}\left(C_{n_{1}}-C_{n_{1}+2 n}\right)}{\lambda_{n}-\left(\pi\left(n+n_{1}\right)\right)^{2}}\right\} \\
+\sum_{\substack{n_{1}, n_{2}=-\infty \\
n_{1}, n_{1}+n_{2} \neq 0,-2 n}}^{\infty} \frac{C_{n_{1}} C_{n_{2}}\left(\Phi_{n}(x), \sin \left(n+n_{1}+n_{2}\right) \pi x\right)}{\lambda_{n}-\left(\pi\left(n+n_{1}\right)\right)^{2}}
\end{gathered}
$$

Applying the above iteration to last sum and isolating the terms for which $n_{1}+n_{2}+n_{3}=0,-2 n$. We obtain

$$
\begin{gathered}
\left(\lambda_{n}-(\pi n)^{2}\right)\left(\Phi_{n}(x), \sin n \pi x\right)=\left(\Phi_{n}(x), \sin n \pi x\right)\left\{-C_{2 n}\right. \\
+\sum_{\substack{n_{1}=-\infty \\
n_{1} \neq 0,-2 n}}^{\infty} \frac{C_{n_{1}}\left(C_{n_{1}}-C_{n_{1}+2 n}\right)}{\lambda_{n}-\left(\pi\left(n+n_{1}\right)\right)^{2}} \\
\left.+\sum_{\substack{n_{1}, n_{2}=-\infty \\
n_{1}, n_{1}+n_{2} \neq 0,-2 n}}^{\infty} \frac{C_{n_{1}} C_{n_{2}}\left(C_{n_{1}+n_{2}}-C_{n_{1}+n_{2}+2 n}\right)}{\left[\lambda_{n}-\left(\pi\left(n+n_{1}\right)\right)^{2}\right]\left[\lambda_{n}-\left(\pi\left(n+n_{1}+n_{2}\right)\right)^{2}\right]}\right\} \\
+\sum_{\substack{n_{1}, n_{2}, n_{3}=-\infty \\
n_{1}, n_{1}+n_{2}, n_{1}+n_{2}+n_{3} \neq 0,-2 n}}^{\infty} \frac{C_{n_{1}} C_{n_{2}} C_{n_{3}}\left(\Phi_{n}(x), \sin \left(n+n_{1}+n_{2}+n_{3}\right) \pi x\right)}{\left[\lambda_{n}-\left(\pi\left(n+n_{1}\right)\right)^{2}\right]\left[\lambda_{n}-\left(\pi\left(n+n_{1}+n_{2}\right)\right)^{2}\right]}
\end{gathered}
$$

Repeating this process $m$ times we get

$$
\left(\lambda_{n}-(\pi n)^{2}-A_{m}\left(\lambda_{n}\right)\right)\left(\Phi_{n}(x), \sin n \pi x\right)=R_{m+1}
$$

where

$$
\begin{gathered}
A_{m}\left(\lambda_{n}\right)=\sum_{k=0}^{m} a_{k}\left(\lambda_{n}\right), \\
a_{0}\left(\lambda_{n}\right)=-C_{2 n}, \\
a_{1}\left(\lambda_{n}\right)=\sum_{\substack{n_{1}=-\infty \\
n_{1} \neq 0,-2 n}}^{\infty} \frac{C_{n_{1}}\left(C_{n_{1}}-C_{n_{1}+2 n}\right)}{\lambda_{n}-\left(\pi\left(n+n_{1}\right)\right)^{2}}, \\
a_{2}\left(\lambda_{n}\right)=\sum_{\substack{n_{1}, n_{2}=-\infty \\
n_{1}, n_{1}+n_{2} \neq 0,-2 n}}^{\infty} \frac{C_{n_{1}} C_{n_{2}}\left(C_{n_{1}+n_{2}}-C_{n_{1}+n_{2}+2 n}\right)}{\left[\lambda_{n}-\left(\pi\left(n+n_{1}\right)\right)^{2}\right]\left[\lambda_{n}-\left(\pi\left(n+n_{1}+n_{2}\right)\right)^{2}\right]}
\end{gathered}
$$

and in general for $k$

$$
a_{k}\left(\lambda_{n}\right)=\sum_{n_{1}, n_{2}, \ldots, n_{k}=-\infty}^{\infty} \frac{C_{n_{1}} C_{n_{2}} \ldots C_{n_{k}}\left(C_{n_{1}+n_{2}+. .+n_{k}}-C_{n_{1}+n_{2}+\ldots+n_{k}+2 n}\right)}{\prod_{j=1}^{k}\left[\lambda_{n}-\left(\pi\left(n+\sum_{s=1}^{j} n_{s}\right)\right)^{2}\right]}
$$




$$
R_{m+1}=\sum_{n_{1}, n_{2}, \ldots, n_{m+1}=-\infty}^{\infty} \frac{C_{n_{1}} C_{n_{2}} \ldots C_{n_{m+1}}\left(q(x) \Phi_{n}(x), \sin \left(n+\sum_{k=1}^{m+1} n_{k}\right)\right)}{\prod_{j=1}^{m+1}\left[\lambda_{n}-\left(\pi\left(n+\sum_{k=1}^{j} n_{k}\right)\right)^{2}\right]},
$$

Here the sums for $a_{k}\left(\lambda_{n}\right)$ and $R_{m+1}$ are taken under the conditions

$$
\sum_{j=1}^{s} n_{j} \neq 0,-2 n,
$$

for $s=1,2, \ldots, k$ and $s=1,2, \ldots, m+1$ respectively. Moreover in obtaining (17) after $m^{\text {th }}$ iteration we applied (5). Using (4) and arguing as in the proof of equalities (27), (28) of [2] we get the following relations

$$
\begin{gathered}
\sum_{\substack{k=-\infty \\
k \neq-n, n}}^{\infty} \frac{1}{\left|\lambda_{n}-(\pi k)^{2}\right|}=O\left(\frac{\ln |n|}{n}\right), \\
a_{k}=O\left(\left(\frac{\ln |n|}{n}\right)^{k}\right), R_{m+1}=O\left(\left(\frac{\ln |n|}{n}\right)^{m+1}\right) .
\end{gathered}
$$

Theorem 2.1. The eigenvalue $\lambda_{n}$ of the operator $L(q)$ satisfies the asymptotic formula

$$
\lambda_{n}=(\pi n)^{2}+F_{m}+O\left(\left(\frac{\ln |n|}{n}\right)^{m+1}\right),
$$

for all $m=0,1,2, \ldots$, where $F_{0}=-C_{2 n}$,

$$
\begin{gathered}
F_{1}=A_{1}\left((n \pi)^{2}\right)=-C_{2 n}+\sum_{\substack{n_{1}=-\infty \\
n_{1} \neq-2 n}}^{\infty} \frac{C_{n_{1}}\left(C_{n_{1}}-C_{n_{1}+2 n}\right)}{\left[(n \pi)^{2}-\left(\pi\left(n+n_{1}\right)\right)^{2}\right]} \\
F_{k}=A_{k}\left((n \pi)^{2}+F_{k-1}\right), \forall k=2,3, \ldots
\end{gathered}
$$

Proof: We shall use induction to prove this theorem. First we prove (20) for $m=0$, that is,

$$
\lambda_{n}=(\pi n)^{2}-C_{2 n}+O\left(\frac{\ln n}{n}\right),
$$

Using the well-known formula (see[7,p.77])

$$
\Phi_{n}(x)=\sqrt{2} \sin n \pi x+O\left(\frac{1}{n}\right)
$$

we get

$$
\left(\Phi_{n}(x), \sin n \pi x\right)=\frac{\sqrt{2}}{2}+O\left(\frac{1}{n}\right)
$$

Therefore dividing both sides of (14) for $m=0$ by $\left(\Phi_{n}(x), \sin n \pi x\right)$ and using (19), (22) we get the proof of (21).

Now we prove (20) $m=1$. Arguing as we have done in the proof of (20) we get from (14) for $m=1$.

$$
\lambda_{n}=(n \pi)^{2}+A_{1}\left(\lambda_{n}\right)+O\left(\left(\frac{\ln |n|}{n}\right)^{2}\right)
$$

Using $\lambda_{n}-(n \pi)^{2}=O(1)$ and (18) one can easily verify that

$$
\sum_{\substack{k=-\infty \\ k_{2} \neq n,-n}}^{\infty}\left|\frac{1}{\lambda_{n}-(\pi k)^{2}}-\frac{1}{(\pi n)^{2}-(\pi k)^{2}}\right|=O\left(\left(\frac{\ln |n|}{n}\right)^{2}\right)
$$


and hence

$$
A_{1}\left(\lambda_{n}\right)=A_{1}\left((\pi n)^{2}\right)+O\left(\left(\frac{\ln |n|}{n}\right)^{2}\right)
$$

This together with (23) proves (20) for $m=1$

In order to prove (20) by induction, we assume it to be true for $m=j-1$, that is,

$$
\lambda_{n}=(n \pi)^{2}+F_{j-1}+O\left(\left(\frac{\ln |n|}{n}\right)^{j}\right)
$$

Replacing $m$ by $j$ in (14), dividing both sides by $\left(\Phi_{n}(x), \sin n \pi x\right)$ and use (19), (22) we get

$$
\lambda_{n}=(n \pi)^{2}+A_{j}\left(\lambda_{n}\right)+O\left(\left(\frac{\ln |n|}{n}\right)^{j+1}\right)
$$

Substituting the value of $\lambda_{n}$ given by (26) in $A_{j}\left(\lambda_{n}\right)$, and arguing as in the proof of (25) we see that

$$
\begin{gathered}
A_{j}\left(\lambda_{n}\right)=A_{j}\left((n \pi)^{2}+F_{j-1}+O\left(\left(\frac{\ln |n|}{n}\right)^{j}\right)\right) \\
=A_{j}\left((n \pi)^{2}+F_{j-1}\right)+O\left(\left(\frac{\ln |n|}{n}\right)^{j+1}\right)
\end{gathered}
$$

Thus taking into account that $A_{j}\left((n \pi)^{2}+F_{j-1}\right)=F_{j}$ we get the proof of (20) for $m=j$ from (27), (28). The theorem is proved.

As an example for asymptotic formulas (20), let the potential $q(x)$ of the operator $L(q)$ with the concrete potential

$$
q(x)=\frac{c}{x^{\alpha}}, \quad 0<\alpha<1
$$

where $c$ is a complex number.

Theorem 2.2. The eigenvalues of the operator $L(q)$ with the potential (29) satisfy the formulas

$$
\lambda_{n}=(n \pi)^{2}-\frac{c . d}{(2 n)^{1-\alpha}}+O\left(\frac{1}{n}\right)
$$

where $d=\int_{0}^{\infty} \frac{\cos \pi t}{t^{\alpha}} d t$

$$
\lambda_{n}=(n \pi)^{2}-\frac{c . d}{(2 n)^{1-\alpha}}+\sum_{\substack{k=-\infty \\ k \neq 0,-2 n}}^{\infty} \frac{f_{k}\left(f_{k}-f_{k+2 n}\right)}{(n \pi)^{2}-(\pi(n+k))^{2}}+O\left(\left(\frac{\ln |n|}{n}\right)^{2}\right)
$$

where

$$
f_{n}=\int_{0}^{1} \frac{c \cdot \cos \pi n x}{x^{\alpha}} d x
$$

This theorem is as proved in [1]

\section{Numerical Approximation}

Now, we consider the small eigenvalues of the $L(q)$ operator by numerical method.

For the finite difference method, take an equally spaced mesh $(m \geqslant 2), 0=x_{0}<x_{1}<\ldots<x_{m+1}=1$ where $x_{j}=j h, h=\frac{1}{m+1}$. Writing $y\left(x_{j}\right)$ as $w_{j}, q\left(x_{j}\right)$ as $q_{j}$ and $y^{\prime \prime}\left(x_{j}\right)$ as $w_{j}^{\prime \prime}$, we use the centred difference approximation $-w_{j}^{\prime \prime} \approx \frac{-w_{j-1}+2 w_{j}-w_{j+1}}{h^{2}}$ Substituting in (1) gives the approximating scheme

$$
\frac{-w_{j-1}+2 w_{j}-w_{j+1}}{h^{2}}+q_{j} w_{j}=\Lambda w_{j} \quad j=1,2, \ldots, m .
$$


to solve this problem numericaly .

We should use centered approximation to obtain these values. After the calculation this can be written in matrix form as the order $(m)$ system

$$
(K+Q) w=\Lambda^{m} w
$$

where $K$ is a tridiagonal matrix, $Q=\operatorname{diag}\left(q\left(x_{1}\right), q\left(x_{1}\right), \ldots q\left(x_{m}\right)\right)$ and

$$
K=\left(\begin{array}{ccccc}
\frac{2}{h^{2}} & \frac{-1}{h^{2}} & & & \\
-\frac{1}{h^{2}} & \frac{2}{h^{2}} & -\frac{1}{h^{2}} & & \\
& & \cdot & & \\
& & \cdot & & \\
& & -\frac{1}{h^{2}} & \frac{2}{h^{2}} & -\frac{1}{h^{2}} \\
& & & \frac{-1}{h^{2}} & \frac{2}{h^{2}}
\end{array}\right)
$$

The eigenvalues of (1-2) are approximated by the eigenvalues of the matrix $K$. Paine et al.in $[13,20]$ showed that the leading term in the asymptotic expansion of the error for the three-point scheme does not depend on potential $q$. Moreover for such method the error is known in closed form when $q(x)=0$. They therefore suggested to use this information to correct the computed eigenvalue in the case of $q$. With the ordered eigenvalues of (10) denoted by $\Lambda_{k}^{m}, k=1,2, \ldots, m$, the corrected eigenvalue estimates are given by

$$
\bar{\Lambda}_{k}^{(m)}=\Lambda_{k}^{(m)}+\mu_{k}-\mu_{k}^{(m)}, k=1,2, \ldots, m-1
$$

where $\mu_{k}$, and $\mu_{k}^{(m)}=1,2, \ldots$ are the $k$ th exact and numerical eigenvalues for $q=0$, respectively. and $\Lambda_{k}^{(m)}, k=$ $1,2, \ldots m$, are the eigenvalues of (10) with $Q \neq 0$. We apply this technique with $m \gg 1$ to the eigenvalue problem (1-2) then the results given in tables show that the errors in the corrected estimates and superior to the original estimates.

Clearly, the obtained eigenvalues from the formula obtained by the asymptotic method are listed and evaluated numerically the compared corrected eigenvalues.

$\begin{array}{llccr}\text { Table } 1 & \alpha=0.1 & \alpha=0.5 & \alpha=0.9 & \\ n & \lambda_{n}^{(0.1)} & \lambda_{n}^{(0.5)} & \lambda_{n}^{(0.9)} & (n \pi)^{2} \\ 1 & 9,836802 & 9,284288 & -0,82713 & 9,869604 \\ 2 & 39,46010 & 38,98940 & 26,81010 & 39,47842 \\ 3 & 88,81315 & 88,36869 & 74,39831 & 88,82644 \\ 4 & 157,903 & 157,4696 & 141,9462 & 157,9137 \\ 5 & 246,7309 & 246,3026 & 229,4107 & 246,7401 \\ 6 & 355,2976 & 354,8715 & 336,7566 & 355,3058 \\ 7 & 483,6033 & 483,1776 & 463,9588 & 483,6106 \\ 8 & 631,6479 & 631,2219 & 611,0016 & 631,6547 \\ 9 & 799,4316 & 799,0048 & 777,8811 & 799,4380 \\ 10 & 986,9544 & 986,527 & 964,6524 & 986,9604 \\ 20 & 3947,837 & 3947,403 & 3921,531 & 3947,842 \\ 30 & 8882,64 & 8882,196 & 8853,956 & 8882,644 \\ 40 & 15791,36 & 15790,91 & 15760,9 & 15791,37 \\ 50 & 24674,01 & 24673,55 & 24642,1 & 24674,01 \\ 60 & 35530,57 & 35530,1 & 35497,45 & 35530,58 \\ 70 & 48361,06 & 48360,58 & 48326,88 & 48361,06 \\ 80 & 63165,46 & 63164,98 & 63130,34 & 63165,47 \\ 90 & 79943,79 & 79943,3 & 79907,83 & 79943,80 \\ 100 & 98696,04 & 98695,55 & 98659,3 & 98696,04\end{array}$

Table 1 shows both the $n^{\text {th }}$ eigenvalue obtained by using the asymptotic method for $\alpha=0.1,0.5,0.9$ respectively and the $n^{\text {th }}$ eigenvalue for the operator $L(0)$. 
Table 2

$\begin{array}{llll}n & \bar{\Lambda}_{n}^{(0.1)} & \bar{\Lambda}_{n}^{(0.5)} & \bar{\Lambda}_{n}^{(0.9)} \\ 1 & 10,95108 & 11,37728 & 12,03832 \\ 2 & 40,57302 & 41,12744 & 42,17357 \\ 3 & 89,92594 & 90,53919 & 91,81482 \\ 4 & 159,0158 & 159,6646 & 161,1022 \\ 5 & 247,8438 & 248,5171 & 250,0792 \\ 6 & 356,4106 & 357,102 & 358,765 \\ 7 & 484,7162 & 485,4218 & 487,1692 \\ 8 & 632,7609 & 633,478 & 635,2977 \\ 9 & 800,5447 & 801,2712 & 803,1541 \\ 10 & 988,0675 & 988,8021 & 990,741 \\ 20 & 3948,951 & 3949,728 & 3952,013 \\ 30 & 8883,753 & 8884,548 & 8887,002 \\ 40 & 15792,48 & 15793,28 & 15795,82 \\ 50 & 24675,12 & 24675,93 & 24678,49 \\ 60 & 35531,69 & 35532,49 & 35535,03 \\ 70 & 48362,17 & 48362,97 & 48365,43 \\ 80 & 63166,58 & 63167,36 & 63169,66 \\ 90 & 79944,9 & 79945,64 & 79947,63 \\ 100 & 98697,13 & 98697,56 & 98698,28\end{array}$

Table 2 shows the $n^{\text {th }}$ corrected eigenvalue obtained by using the finite difference method for $\alpha=0.1,0.5,0.9$ respectively.

$\begin{array}{llll}\text { Table } 3 & & & \\ n & \bar{\Lambda}_{n}^{(0.1)}-(n \pi)^{2} & \bar{\Lambda}_{n}^{(0.5)}-(n \pi)^{2} & \bar{\Lambda}_{n}^{(0.9)}-(n \pi)^{2} \\ 1 & 1,081479893 & 1,507673921 & 2,16871913 \\ 2 & 1,094606645 & 1,649021921 & 2,695156874 \\ 3 & 1,09950304 & 1,712745752 & 2,988379079 \\ 4 & 1,102092404 & 1,750905378 & 3,188503476 \\ 5 & 1,103703936 & 1,776991574 & 3,339132542 \\ 6 & 1,104807505 & 1,796256532 & 3,459199448 \\ 7 & 1,105612449 & 1,811223831 & 3,558567804 \\ 8 & 1,106226483 & 1,823276353 & 3,643007064 \\ 9 & 1,106710823 & 1,833243483 & 3,71617449 \\ 10 & 1,107102891 & 1,841656869 & 3,780529819 \\ 20 & 1,108918458 & 1,886274806 & 4,171554409 \\ 30 & 1,109516903 & 1,904096257 & 4,358259832 \\ 40 & 1,109768063 & 1,912303842 & 4,45191185 \\ 50 & 1,109843865 & 1,914880513 & 4,482477541 \\ 60 & 1,109782116 & 1,912764558 & 4,457511461 \\ 70 & 1,109552451 & 1,905194836 & 4,370946909 \\ 80 & 1,109004827 & 1,888648163 & 4,196242546 \\ 90 & 1,10742319 & 1,848625597 & 3,838723497 \\ 100 & 1,081623478 & 1,515892515 & 2,237212549\end{array}$

The corrected differences $\bar{\Lambda}_{n}^{(\alpha)}-(n \pi)^{2}$ show the effect of the potential $q^{(\alpha)}$ to the eigenavlues for $\alpha=0.1,0.5,0.9$ 


$\begin{array}{llll}\text { Table } 4 & & & \\ n & \lambda_{n}^{(0.1)}-(n \pi)^{2} & \lambda_{n}^{(0.5)}-(n \pi)^{2} & \lambda_{n}^{(0.9)}-(n \pi)^{2} \\ 1 & -0,032802560 & 0,585315946 & -10,69673415 \\ 2 & -0,018317047 & -0,489017416 & -12,66831515 \\ 3 & -0,013287336 & -0,457752089 & -14,42813566 \\ 4 & -0,010719567 & -0,444067713 & -15,96747315 \\ 5 & -0,009160065 & -0,437461285 & -17,32939883 \\ 6 & -0,008112923 & -0,434302424 & -18,54919126 \\ 7 & -0,007362049 & -0,433014772 & -19,65180001 \\ 8 & -0,006797985 & -0,432800998 & -20,65304927 \\ 9 & -0,006359217 & -0,433180466 & -21,55685193 \\ 10 & -0,006007514 & -0,433478708 & -22,30805135 \\ 20 & -0,004445580 & -0,438827058 & -26,31055883 \\ 30 & -0,003965214 & -0,448262049 & -28,68837815 \\ 40 & -0,003751163 & -0,457500814 & -30,46864132 \\ 50 & -0,003640011 & -0,465942480 & -31,90956508 \\ 60 & -0,003578065 & -0,473584198 & -33,12753648 \\ 70 & -0,003542804 & -0,480523723 & -34,18640325 \\ 80 & -0,003523263 & -0,486863986 & -35,12538671 \\ 90 & -0,003513533 & -0,492694053 & -35,97046725 \\ 90 & -0,003510185 & -0,498087369 & -36,73981629\end{array}$

The differences $\lambda_{n}^{(\alpha)}-(n \pi)^{2}$ show the effect of the potential $q^{(\alpha)}$ to the eigenavlues for $\alpha=0.1,0.5,0.9$.

\section{Conclusion}

It is natural and well known that for large eigenvalues the asymptotic method gives us approximations with smaller errors. The numerical method, in general, gives better results for smaller eigenvalues. The Tables 1,2 show that the results of both the asymptotic method and corrected method give quiet acceptable results for eigenvalues. According to the Table 3 and 4, if $\alpha$ increases, the effect of the potential function to the eigenvalues also increases for both methods.

Therefore we can easily observe that the correcting method and the asymptotic method give good results for the calculation of the eigenvalues. It is natural and well known that for large eigenvalues the asymptotic method gives us approximations with smaller errors. The numerical method, in general, gives better results for smaller eigenvalues. We see that in this paper corrected eigenvalues also give us approximations with smaller errors for all eigenvalues.

The table 3 and 4 show that the results of the asymptotic method and numerical method also give quiet acceptable results for $\alpha=0.1$. Therefore we can easily observe that asymptotic and corrected methods give good results for the calculation of all eigenvalues. Additionally while $\alpha$ tends to 1, both of the methods are effected by potential function and differences between two methods are also increasing.

\section{Acknowledgment}

This work was supported by Research Fund of the Marmara University. Project Number: 6242

\section{References}

[1] B. Yilmaz and O. A. Veliev, "Asymptotic formulas for Dirichlet boundary value problems," Studia Scientiarum Mathematicarum Hungarica, vol. 42, no. 2, pp. 153-171, 2005.

[2] O. A. Veliev and M. Toppamuk Duman, "The spectral expansion for a nonself-adjoint Hill operator with a locally integrable potential," Journal of Mathematical Analysis and Applications, vol. 265, no. 1, pp. 76-90, 2002.

[3] G. D. Birkhoff, "Boundary value and expansion problems of ordinary linear differential equations," Transactions of the AmericanMathematical Society, vol. 9, no. 4, pp. 373-395, 1908. 
[4] N. Dunford and J. T. Schwartz, Linear Operators. Part III, Spectral Operators, Wiley-Interscience, New York, NY, USA, 1988.

[5] W. N. Everitt, J. Gunson, and A. Zettl, "Some comments on Sturm-Liouville eigenvalue problems with interior singularities,"Journal of Applied Mathematics and Physics, vol. 38, no. 6, pp. 813-838, 1987.

[6] V. A. Marchenko, Sturm-Liouville Operators and Applications, Birkh“auser, Basel, Switzerland, 1986.

[7] M. A.Naimark, Linear Differential Operators, GeorgeG. Harrap and Company, 4th edition, 1967.

[8] B. N. Parlett,The Symmetric Eigenvalue Problem, Prentice-Hall, Englewood Cliffs, NJ, USA, 1980.

[9] J. D. Tamarkin, "Some general problems of the theory of ordinary linear differential equations and expansion of an arbitrary function in series of fundamental functions," Mathematische Zeitschrift, vol. 27, no. 1, pp. 1-54, 1928.

[10] E. C. Titchmarsh, Eigenfunction Expansions, vol. I, Oxford University Press, 1962.

[11] R. L. Burden, Numerical Analysis, Brooks Cole, Pacific Grove,

[12] A. L. Andrew, "Correction of finite difference eigenvalues of periodic Sturm-Liouville problems," Australian Mathematical Society Journal Series B, vol. 30, no. 4, pp. 460-469, 1989.

[13] J. W. Paine, F. R. de Hoog, and R. S. Anderssen, "On the correction of finite difference eigenvalue approximations for Sturm-Liouville problems," Computing, vol. 26, no. 2, pp. 123-139, 1981.

[14] R. S. Anderssen and F. R. de Hoog, "On the correction of finite difference eigenvalue approximations for Sturm-Liouville problems with general boundary conditions," BIT, vol. 24, no. 4, pp. 401-412, 1984.

[15] A. L. Andrew and J. W. Paine, "Correction of finite element estimates for Sturm-Liouville eigenvalues," Numerische Mathematik, vol. 50, no. 2, pp. 205-215, 1986.

[16] C.-K. Chen and S.-H. Ho, "Application of differential transformation to eigenvalue problems," Applied Mathematics and Computation, vol. 79, no. 2-3, pp. 173-188, 1996.

[17] P. Ghelardoni, "Approximations of Sturm-Liouville eigenvalues using boundary value methods," Applied Numerical Mathematics, vol. 23, no. 3, pp. 311-325, 1997.

[18] P. Ghelardoni and G. Gheri, "Improved shooting technique for numerical computations of eigenvalues in Sturm-Liouville problems," Nonlinear Analysis, vol. 47, pp. 885-896, 2001.

[19] J. D. Pryce, Numerical Solution of Sturm-Liouville Problems, Clarendon Press, Oxford, UK, 1993.

[20] B.Yilmaz, Guldem Yildiz „, O. A. Veliev, "Asymptotic and Numerical Methods in Estimating Eigenvalues", Mathematical Problems in Engineering , 2013

\section{Affiliations}

\section{BÜLENT YILMAZ}

AdDRESS: Marmara University, Dept. of Mathematics, 34722, Istanbul-Turkey.

E-MAIL: bulentyilmaz@marmara.edu.tr 